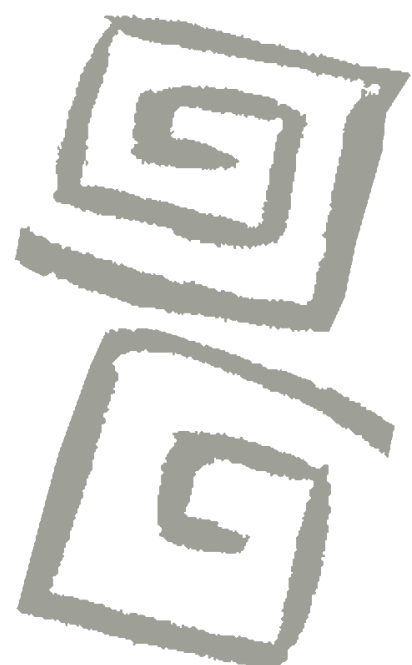

\title{
Antropología médica en América Latina 1990-2015: Una revisión estrictamente provisional
}

\author{
Medical anthropology in Latin America, 1990-2015: A \\ strictly provisional review
}

Eduardo L. Menéndez ${ }^{1}$

${ }^{1}$ Doctor en Ciencias Antropológicas. Profesorinvestigador emérito Centro de Investigaciones y Estudios Superiores en Antropología Social (CIESAS). Coordinador, Seminario Permanente de Antropología Médica (SPAM), México. $\triangle$ iD
RESUMEN Se presenta una aproximación provisional sobre lo producido por la antropología médica en América Latina, especialmente, en México y, en menor medida, en Brasil, entre 1990 y 2015, tratando de señalar sus principales aportes, orientaciones y objetivos, pero también planteando críticas y algunas dudas referidas, especialmente, a la omisión del estudio de graves procesos y problemas de salud colectiva. En el artículo se trata de poner en evidencia la importancia de esta disciplina no solo para la antropología, sino también para la biomedicina, planteando la necesidad de complementación, más allá de las diferencias e incompatibilidades que existen respecto, por ejemplo, del uso de las técnicas cualitativas o de las intervenciones y no intervenciones sobre los "usos y costumbres" de los diferentes actores sociales referidos a los procesos de salud/enfermedad/ atención-prevención.

PALABRAS CLAVES Antropología Médica; Biomedicina; Metodología.

ABSTRACT The article presents a provisional examination of the production of Latin American medical anthropology, especially from Mexico, and to a lesser degree Brazil, from 1990 to 2015, in an attempt to highlight the discipline's principal contributions, orientations and objectives, but also to pose critiques and doubts, especially with respect to the omission of the study of serious collective health problems and processes. The article attempts to put into evidence the importance of the discipline not only for anthropology, but also for biomedicine, suggesting the need for complementation beyond the differences and incompatibilities that exist regarding, for example, the use of qualitative techniques, and interventions -or lack of interventions- regarding the customs of the different social actors with respect to health/disease/care-prevention processes.

KEY WORDS Medical Anthropology; Biomedicine; Methodology. 


\section{UNA PROPUESTA PROVISIONAL}

En este texto voy a reflexionar sobre la situación de la antropología médica generada en América Latina entre 1990 y la actualidad, buscando establecer tentativamente cuáles son las temáticas más estudiadas, proponiendo algunas tendencias generales, para luego cuestionar ciertos aspectos, y concluir señalando varios de sus aportes y planteando algunas dudas. Considero que el nombre de antropología médica no es el más apropiado, sin embargo, los otros como antropología de la salud, antropología de la medicina o etnomedicina son aún menos satisfactorios por sus connotaciones ideológicas, por su incongruencia o por su especificidad. Por lo cual, mantenemos este nombre, pese a las críticas, simplemente porque es el de mayor uso entre nosotros.

Si bien pretendo referirme a América Latina, mi texto trata sobre todo de México y en menor medida de Brasil, permaneciendo en un segundo plano el resto de los países latinoamericanos, subrayando que lo que voy a proponer constituye una aproximación provisional.

Partimos de reconocer un desarrollo diferencial de la antropología médica según países en América Latina. Así, por ejemplo, si tomo los dos países con mayor producción y diversidad actual de esta disciplina en la región, encuentro que en México hay un desarrollo sostenido de estudios sobre procesos de salud/enfermedad/atención-prevención (de ahora en adelante procesos de s/e/a-p) referido a los pueblos denominados originarios, por lo menos desde la década de 1930, que se afirma en la de 1940 y 1950, continuando hasta la actualidad a través de modificaciones sustantivas desarrolladas, sobre todo, desde finales de la década de 1970. Pero asumiendo, además, que desde la década de 1930 también trabajaron en México algunos de los más importantes antropólogos médicos estadounidenses, que difundieron tempranamente las orientaciones dominantes en este campo, incluyendo no solo producción etnográfica, sino propuestas teóricas que parecen desconocer los antropólogos actuales o, lo que puede ser más grave, tachándolas de meramente empíricas.

Pero recordando que, en gran parte de estos estudios se genera, si no una ruptura, por lo menos un distanciamiento y una reformulación de la relación entre los procesos de s/e/a-p y los procesos mágico-religiosos, que constituye uno de los puntos básicos del desarrollo de una antropología médica más o menos autónoma. Si bien la ruptura con la dimensión mágico-religiosa de la enfermedad y la cura, tuvo consecuencias positivas para poder recuperar las dimensiones del sufrimiento, de la mortalidad, de la enfermedad, que eran totalmente opacadas por los estudios de los procesos mágico-religiosos, sin embargo, condujo a reducir cada vez más su papel en el proceso de s/e/a-p. Lo óptimo sería articular las dos dimensiones.

Desde esta perspectiva, en México se genera un interés etnográfico, teórico y aplicado sobre este campo mucho antes que en la mayoría de las antropologías latinoamericanas y europeas, que en gran medida tiene que ver con los intereses y objetivos no solo del indigenismo inicial, sino con las corrientes teóricas que se desarrollaron ulteriormente, y que estaban relacionadas con los proyectos contradictorios emergidos de la "revolución mexicana" ${ }^{\prime \prime 1,2,3,4)}$.

Mientras que, en el caso de Brasil, como reconocen las propias especialistas brasileñas ${ }^{(5,6,7,8)}$, la situación de la antropología médica fue débil hasta la década de 1980, generándose sobre todo, desde 1990, un notable desarrollo que en gran medida tiene que ver con sus vinculaciones con el movimiento de salud colectiva, que incluye la creación en Brasil en estos años de un servicio de atención médica diferenciado para los grupos nativos ${ }^{(9,10)}$.

También, en el caso de México, hubo tempranas relaciones con la salud pública y la medicina social, así como con las organizaciones no gubernamentales (ONG) dedicadas a los procesos de s/e/a-p; pero dichas vinculaciones se dieron hasta fechas recientes en términos personales o microgrupales, más que institucionales. Necesitamos asumir 
que, desde las décadas de 1970 y 1980 se gestó en varios países latinoamericanos un notable desarrollo de ONG que trabajaron sobre procesos de s/e/a-p, que en gran medida manejaron enfoques antropológicos.

Ahora, si bien la antropología cultural de EEUU influyó notoriamente desde 1930 en los estudios sobre los procesos de s/e/a$\mathrm{p}$ realizados en América Latina, lo hizo en forma conflictiva y discontinuada, sobre todo a partir de 1960, ya que si bien algunas tendencias, como la representada por Aguirre Beltrán ${ }^{(1,11)}$ adhieren -aunque críticamente- a las orientaciones estadounidenses de tipo funcionalista/culturalista, otras -como la impulsada por Bonfil BataIla ${ }^{(12)}$ - las cuestionaron planteando alternativas y diferencias que se articulaban con las tendencias críticas que se estaban dando en nuestros países a nivel de la antropología social y que, a través de la obra de autores como Darcy Ribeiro o Pablo González Casanova, cuestionaban el colonialismo no solo político, sino también el integrado en las propuestas antropológicas.

$Y$ recordando que una tendencia fuerte de las corrientes latinoamericanas durante este lapso fue el cuestionamiento al "psicologismo" de una parte de la antropología estadounidense, y a una correlativa exclusión del sujeto y su subjetividad, lo cual dominaba en el conjunto de las ciencias sociales y sobre todo en las que adherían a propuestas políticas e ideológicas de "izquierda"(13). Además, las bases formativas de los antropólogos latinoamericanos que estudiaron en las facultades de humanidades entre las décadas de 1940 y 1960 referían a corrientes teóricas que diferían de las dominantes en EEUU, de tal manera que, tanto las corrientes neokantianas e historicistas y especialmente Dilthey, como las fenomenológicas y existencialistas estaban en la base de la formación, por lo menos en algunos de nuestros países.

Es además importante consignar que, a nivel latinoamericano, incluidos los dos países señalados, la antropología médica fue considerada, desde sus inicios, por los dueños teóricos de la antropología social y de la etnología como una especialidad "técnica", empírica, aplicada e incluso ateórica; lo que se mantiene hasta la actualidad. Al respecto, es interesante consignar que cuando en 2009 surgió en México la epidemia de influenza A (H1N1), durante las tres primeras semanas los periódicos se inundaron de artículos escritos por numerosos intelectuales, incluidos científicos sociales, quienes previamente no habían tenido que ver con el estudio o con reflexiones sobre los procesos de s/e/a-p, pero que ahora encontraban una nueva oportunidad para "teorizar"(14).

El cuerpo central de la antropología médica en América Latina, y más allá de las orientaciones teóricas y metodológicas dominantes y no dominantes, necesita ser relacionado con la situación socieconómica y sociopolítica de nuestros países, y con los momentos históricos dentro de los cuales se desarrollaron. Y, por lo tanto, en nuestro caso necesita ser relacionado con el proceso de descolonización gestado entre 1940 y 1960 en el denominado Tercer Mundo, con el paso a primer plano de los populismos nacionalistas, así como con las políticas desarrollistas, y desde la década de 1980 con las neoliberales y "neopopulistas" que corresponden al lapso que estamos analizando, pero que solo enumeramos, ya que no podemos profundizar su estudio en esta oportunidad.

La oscilación entre estas orientaciones económico/políticas posibilitó el desarrollo de corrientes políticas y teóricas que plantearon tanto la cuestión de la "diferencia" cultural y el empoderamiento de los pueblos originarios, como el desarrollo de los estudios de género y las luchas por la situación de la mujer. Y si bien bajo los gobiernos "neopopulistas" se redujo la pobreza y en países como Bolivia y Ecuador se fortalecieron los movimientos étnicos, la tendencia dominante en la región ha sido la del incremento de las desigualdades socioeconómicas.

Pero, además, el desarrollo de la antropología médica latinoamericana debe ser relacionado con la situación social de los pueblos amerindios, cruelmente expresada en que estos pueblos han sido y siguen siendo el sector social con las mayores tasas de mortalidad 
general y por grupos etarios, y con la menor esperanza de vida ${ }^{(15,16,17,18,19)}$. Como señalan Langdon y Cardoso:

Más allá del tamaño de la población y con base en datos epidemiológicos incompletos, todos estos países [de América Latina] presentan tasas que señalan una situación de salud peor para los pueblos indígenas en comparación con las de la sociedad circundante. Lo que los distintos países tienen en común en ese escenario son las altas tasas de enfermedades endémicas, carenciales y crónicas, que muestran las violencias estructurales que impactan en sus vidas tal como fue observado, en otro contexto, por Farmer [...] En todos estos países, los indígenas sufren tasas más altas de desnutrición, mortalidad infantil, abuso de substancias, suicidio y otras. Los problemas de las enfermedades crónicas, tales como hipertensión y diabetes reflejan los profundos cambios ambientales, territoriales y de estrategias de subsistencia. La situación sanitaria de los pueblos indígenas refleja también procesos históricos comunes de subordinación política, explotación económica, discriminación social, posición de marginalidad y la inadecuación de la atención en salud. ${ }^{(20)}$

Y esta situación negativa de salud de dichos pueblos ha sido históricamente marginal a los objetivos del sector salud de nuestros países, más allá de que corrientes como el indigenismo integracionista hayan buscado intervenir a través de concepciones generadas por las teorías de la modernización, o que las corrientes críticas colocaran sus expectativas en cambios radicales del sistema económico/ político, incluido el empoderamiento de los grupos indígenas; y que otras corrientes más recientes piensen en acciones microsociales y subjetivas para reducir o eliminar las aflicciones de los sufrientes.

\section{¿QUÉ ESTUDIA Y QUÉ NO ESTUDIA LA ANTROPOLOGIA MÉDICA EN AMÉRICA LATINA?}

Actualmente, al igual que en EEUU y en países europeos, la antropología médica regional, y sobre todo la de Brasil y México, estudia casi todo proceso de s/e/a-p en muy diferentes sectores y actores sociales, con los objetivos de describirlos y comprenderlos, y en menor grado intervenir sobre ellos. Si bien los actores sociales que ahora estudiamos no se reducen a los grupos étnicos originarios, sin embargo, estos siguen siendo el actor principal en los estudios latinoamericanos.

A partir de la revisión de revistas antropológicas y de salud pública, de programas de congresos nacionales, regionales y latinoamericanos, así como de los escasos trabajos de revisión existentes, codifiqué para el lapso 1990-2015, 152 categorías referidas a procesos, temas y problemas que agrupé en las veinte categorías que enumero a continuación y que aparecen ordenadas en orden alfabético: 1) alimentación/desnutrición; 2) autoatención/grupos de autoayuda/ cuidadores; 3) biomedicina e instituciones biomédicas (incluyendo la industria químico/farmacéutica); 4) cuerpo, subjetividad; 5) curadores tradicionales; 6) emociones, sufrimientos; 7) enfermedades crónico-degenerativas; 8) enfermedades infectocontagiosas (incluye $\mathrm{VIH}$-sida); 9) enfermedades mentales incluidas "adicciones"; 10) enfermedades tradicionales; 11) epidemiología sociocultural; 12) estudios de género y salud; 13) medicinas alternativas y/o complementarias; 14) migración y procesos de s/e/a-p; 15) modelos de atención de la salud, pluralismo médico; 16) poder y procesos de s/e/a-p; 17) políticas públicas y políticas de salud (incluyendo derechos humanos); 18) salud intercultural; 19) salud reproductiva; 20) violencias. Incluyendo dos últimos apartados que corresponden a teoría y metodología. Se recuerda que todas las categorías refieren a procesos de s/e/a-p, estudiados desde las diferentes corrientes que integran la antropología médica. 
Ahora bien, hay una serie de importantes procesos y temáticas que, sin embargo, han tenido un escaso desarrollo entre nosotros, sin negar que existen estudios al respecto. Algunas de esas temáticas son las desigualdades socioeconómicas y los procesos de s/e/a-p; el papel de estos procesos en los movimientos sociales, incluidos los movimientos étnicos; el papel del saber de los curadores tradicionales en las propuestas ideológico-políticas de dichos movimientos; el creciente proceso de mercantilización de los saberes y productos tradicionales referidos a los procesos de s/e/a-p; estudios sobre la eficacia -no solo simbólica- de las terapéuticas tradicionales y biomédicas observadas en términos de mortalidad y discapacidad respecto de los grupos étnicos; la situación negativa de salud de los varones; la carencia de estudios sobre los "cuerpos" racializados y los "cuerpos" de los trabajadores en términos de padecimientos; la escasez de estudios y reflexiones sobre la capacidad y calidad de agencia de los diferentes actores sociales y especialmente de los subalternos, la poca importancia de los padecimientos relacionados con actividades laborales de todo tipo; la corrupción en los sistemas de salud, y la exclusión de los actores y problemas afroamericanos por parte de la antropología médica.

Uno de los procesos y problemas menos estudiados entre nosotros, pese a que operó intensamente en casi todos los países de la región, es el de las políticas de planificación familiar y, especialmente, el de las políticas de esterilización de mujeres en los grupos étnicos, lo cual contrasta con la importancia de los estudios sobre violencia obstétrica. Políticas que se desarrollaron en forma intensiva desde mediados de la década de 1970, y sobre todo durante las décadas de 1980 y 1990, y que persisten hasta la actualidad.

$\mathrm{Si}$ bien algunas problemáticas han tenido un notorio desarrollo en los últimos años, como es el caso de las emociones; observamos, sin embargo, que la mayoría de estos estudios parece ignorar que gran parte de las enfermedades tradicionales serían generadas por emociones, lo cual se expresa inclusive en el nombre de muchos de esos padecimientos como es el caso del coraje, la vergüenza, y el susto o espanto. Más aún, la causal de gran parte de las enfermedades tradicionales tiene que ver con la envidia, y recordando que las brujerías se ejercen por amor, odio o venganza, que el mal de ojo refiere generalmente al deseo del otro, y que la chipilez tiene que ver no solo con el destete, sino con el amamantamiento de otro niño.

Estos padecimientos pueden referir a depresión, ansiedad, angustia, miedo, enojo, deseo, amor y otras "emociones", que evidencian la notable sensibilidad colectiva que los sujetos y grupos amerindios tienen frente a episodios de su vida cotidiana a los que necesitan convertir en padecimientos para ser tratados a nivel familiar y/o con curadores tradicionales. Más aún, una de esas emociones, la envidia, constituye uno de los principales ejes de relaciones negativas especialmente entre sujetos y microgrupos, siendo en términos emic una de las principales causas de enfermedad y tal vez de muerte. $Y$ recordamos que todos estos padeceres exigen la realización de rituales de sanación, por lo que necesitamos asumir que la medicalización de los comportamientos es previa al desarrollo y expansión de la biomedicina, aunque los antropólogos y otros profesionales no lo piensen en estos términos, o directamente no lo piensen.

Al respecto, considero que en los grupos étnicos latinoamericanos domina una "medicalización tradicional" de toda una serie de padecimientos, que se expresa en la gran cantidad de curadores tradicionales, tanto en números absolutos como proporcionales, que existe a nivel de comunidad en comparación con el personal de salud; en la diversidad de curadores tradicionales con los cuales puede contactar la población; y en que gran parte de las enfermedades tradicionales, como ya lo señalamos, remite a comportamientos. Todas las formas de atención, y no solo la biomédica cumplen en sus sociedades diversas funciones sociales, siendo una de ellas la "medicalización" de comportamientos.

Hay además una tendencia a no aplicar algunos de los conceptos centrales de la antropología a determinados procesos de 
s/e/a-p estudiados; y así, por ejemplo, sabemos que América Latina constituye actualmente la región más homicida del planeta. $Y$ pese a que esto ha sido una constante, sin embargo, la mayoría - por no decir la totalidad- de los antropólogos no utiliza el concepto cultura para describir y analizar las agresiones homicidas; e incluso algunos niegan expresamente que la violencia homicida sea parte de nuestras culturas. A nivel teórico y metodológico considero que las violencias homicidas forman parte de nuestras culturas y no solo de nuestras sociedades; recordando que en el caso de algunas violencias son claramente culturales como las "violencias de sangre", el "mal accidente" (infanticidio), o las agresiones físicas entre varones como parte del denominado "machismo" y, obviamente, los feminicidios. Como todo proceso cultural, dichas violencias homicidas deben ser articuladas con procesos económico-políticos e ideológicos dentro de los que operan, así como también pensarlas en términos de subjetividad y de cambio.

Ahora bien, un aspecto que surge de la producción latinoamericana, es que domina actualmente una antropología del illness, habiendo un escaso desarrollo de una antropología del disease, y una débil producción de una antropología del sickness ${ }^{(21)}$. recordando que illness refiere a cómo el sujeto y su grupo perciben, sienten y actúan los padecimientos; disease a cómo el curador percibe, diagnostica y trata la enfermedad, y sicknes propone incluir el illness y el disease dentro de las condiciones económico-políticas y socioculturales en que opera. Es decir, domina una antropología que se centra en el saber, la experiencia, el sufrimiento, la subjetividad, la trayectoria de los sujetos y microgrupos, pero sin incluir -o incluyendo débilmenteen la mayoría de los casos las condiciones económicas, sociales, políticas, ideológicas e incluso culturales dentro de las cuales surgen y se viven dichos saberes y aflicciones, así como la perspectiva y acciones de la biomedicina.

Es una antropología que estudia los sufrimientos, enfermedades y emociones casi exclusivamente en los sujetos que los padecen, excluyendo a los curadores, y basándose además en el punto de vista de un actor específico, trabajando con las representaciones sociales, los discursos, las narrativas, y/o las experiencias, pero no con las prácticas o identificando a estas con las representaciones, discursos y experiencias. Es una antropología de lo que se dice, más que una antropología de lo que se hace.

Esto no pretende negar la calidad y aportes de gran parte de estos estudios, sino señalar ciertas orientaciones dominantes, que considero que necesitamos evaluar para reflexionar sobre lo que se pierde y lo que se gana con el dominio de dichas orientaciones. Reflexión que implica, por lo menos, incluir el papel no solo de los denominados "determinantes sociales" sino también de los "condicionantes culturales", tanto a nivel micro como macrosocial, no como determinantes, sino como factores frecuentemente decisivos. Prefiero utilizar la expresión "condicionantes estructurales", dado que lo determinativo suele excluir los procesos y actores sociales impidiendo o reduciendo la comprensión, ya que lo estructural no siempre impone sus condiciones. $\mathrm{Y}$, además, porque considero que las dimensiones culturales e ideológicas son también condicionantes estructurales.

Necesitamos además seguir trabajando en lo local -como lo hace la inmensa mayoría de los estudios antropológicos-, pero tratando de encontrar lo global en lo local y, de ser posible, tratando de encontrar lo local en lo global. Considero que la "envidia" -y no solo la del pene o de la teta- podemos observarla a través de lo local y de lo global en los diferentes conjuntos sociales, incluidos los grupos étnicos.

La revisión de la producción antropológica regional nos indica que casi exclusivamente se estudian las enfermedades y los padecimientos, pero no la salud. Si bien, desde los enfoques biomédicos se han propuesto diferentes definiciones de salud, todas concluyeron cuestionadas por médicos y no médicos; pero, no obstante, algunos científicos sociales europeos y estadounidenses han intentado no solo definir sino realizar estudios sobre la "salud positiva" como ha sido el 
caso de Antonosky ${ }^{(22)}$ desde hace varias décadas y más recientemente de Fassin ${ }^{(23)}$. Pero en Latinoamérica solo unos pocos autores han intentado estudiar lo que ellos consideran salud positiva, aunque con escaso éxito, lo cual en gran medida es debido a que la salud tiene que ver muy poco con nuestras mortalidades "evitables" y con las condiciones de nuestros servicios oficiales de salud, así como con nuestras preocupaciones ideológicas, y con los procesos económico-políticos dominantes que vivimos, y que afectan negativamente sobre todo a los sectores sociales subalternos.

Desde las situaciones latinoamericanas hay, por lo menos, dos procesos a considerar respecto del estudio posible de la salud. El primero es que los grupos que estudiamos se caracterizan por tener altas tasas de mortalidad, porque gran parte muere o se discapacita por "causas evitables", porque dichos sujetos y grupos no solo sufren los duelos, las separaciones o las desdichas amorosas, sino que también sufren por hambre, por falta de medicamentos básicos, por migrar o por racismo. Porque el conjunto de los sectores sociales tiene miedo de vivir dentro de comunidades y países que tienen las mayores tasas de homicidio a nivel mundial. Lo cual no solo nos lleva a formularnos interrogantes empíricos e intervenciones posibles, sino a la necesidad de generar explicaciones teóricas de, por ejemplo, por qué los latinoamericanos nos asesinamos tanto.

Es decir, los latinoamericanos vivimos dentro de contextos donde lo prioritario e inmediato no pasa por producir salud, sino por describir, explicar y de ser posible solucionar toda una gama de padecimientos, de los cuales una parte muy frecuentemente son fáciles de reducir o eliminar con las tecnologías existentes; mientras que otros implican la necesidad de estudiarlos y pensarlos básicamente en términos de soluciones político-sociales.

Esto no niega la posibilidad de pensar y estudiar la salud por los que lo quieren hacer, pero a partir de asumir que salud -por lo menos para mí- es un concepto básicamente ideológico, que implica la imposibilidad de generalizaciones teóricas y aplicativas. La salud como eliminación total de los padecimientos corresponde a mitos ancestrales, a las propuestas novelísticas de Un mundo feliz más que de 1984, o a las mitologías biomédicas de sujetos congelados en una espera futura de la sanación total.

Lo que han Ilamado salud los autores que insisten en estudiarla, tiene que ver con sus objetivos e intereses respecto de la enfermedad y sus grupos, y no con la salud, por más malabares semánticos que desarroIlen. Lo cual tampoco niega los intentos de promoción de la salud, pero que realmente constituyen prevenciones de enfermedad. $\mathrm{Ni}$ tampoco desconoce que sectores sociales medios y altos de nuestros países busquen producir salud en sus cuerpos, lo que tiene que ver en gran medida con la negación del envejecimiento. Recordando que no solo la enfermedad, sino cada vez más la salud se ha convertido en un objeto de los deseos personales, pero también de la denominada industria de la salud.

Pero reitero, la salud tiene que ver con objetivos ideológicos específicamente referidos a los procesos de s/e/a-p, o con objetivos políticos como ocurrió entre 1930 y 1960 con toda una serie de conceptos formulados por intelectuales y políticos africanos dentro de la teoría de la "negritud", o como está ocurriendo desde hace unos pocos años con el concepto de "buen vivir" propuesto por intelectuales latinoamericanos etnicistas y más o menos decoloniales, las cuales constituyen propuestas que, más allá de lo que ha pasado con ellas, se caracterizan por usar la salud con objetivos ideológicos.

\section{ALGUNAS CRÍTICAS CERCANAS}

Sin negar los significativos aportes de la antropología médica latinoamericana al conocimiento y a las críticas de determinadas problemáticas, incluyendo propuestas de solución y la realización de intervenciones; necesitamos, no obstante, reconocer toda una serie de aspectos cuestionables, para reflexionar sobre esos aportes. 
Una tendencia constante, y a la cual ya hice referencia, es la de excluir o secundarizar ciertas problemáticas, temáticas y actores sociales y, por lo tanto, no voy a insistir en ello. Pero hay otra antigua tendencia que todavía se mantiene, y me refiero a la que a través de las enfermedades tradicionales que sufre la población y atienden los curadores tradicionales, busca (re)construir las cosmovisiones ancestrales. Y lo busca, por lo menos parcialmente, en la vida actual de los grupos étnicos, colocando sus intereses en el pasado y no en los procesos de enfermedad de los que sufre y muere la población. Sin negar -y lo subrayo- la importancia arqueológica, etnohistórica y/o ideológica que puede tener este objetivo, lo cuestionable es que lleva a una parte de los antropólogos a preocuparse más por las realidades arqueologizantes, que por la situación de salud actual de los grupos caracterizada no solo por las aflicciones, sino por las altas tasas de mortalidad, la baja esperanza de vida y los envejecimientos tempranos. Siendo además una de sus consecuencias, la tendencia al estudio de ciertas enfermedades y a la exclusión de otras. Según Imberton:

Una tendencia muy marcada en los estudios antropológicos sobre las enfermedades en el mundo indígena, pero también sobre otros temas, ha sido la de destacar aquellos aspectos considerados propios de su cosmovisión: el ch'ulel y los naguales entre los mayas, han ocupado un lugar privilegiado en esta perspectiva [...] y ha orientado la mirada antropológica hacia este tema, desatendiendo otros. ${ }^{(24)}$

De tal manera que si una persona de una comunidad choll de Chiapas tiene una enfermedad que no implica la "pérdida del alma" -como es el caso de la vergüenza- es posible que el antropólogo no la estudie. Cuadriello y Megchún ${ }^{(25)}$ corroboran lo planteado por Imberton, refiriéndose a una nueva enfermedad denominada "enfermedad de la tierra" que no está relacionada con aspectos ancestrales, y que no ha sido estudiada por los antropólogos. O como concluye Freire ${ }^{(26)}$ respecto de Venezuela, citando a Kelly:

Los estudios antropológicos tienen una marcada preferencia por centrarse en la "cultura tradicional" de los pacientes, así como en adaptaciones con miras a sintonizar el sistema de salud con sus particularidades, ignorando que el mayor peso en la prestación de servicios médicos a pacientes indígenas está en la "cultura de las instituciones". (26)

Pero quiero señalar que, si bien es importante la rehabilitación cultural de los grupos étnicos, los que lo hacen, por ejemplo, excluyendo analizar las causas que están determinando las altas tasas de mortalidad de dichos grupos y las formas actuales de reducirlas que -y lo subrayo- no tienen que ver con las cosmovisiones autóctonas, tendrían que especificar qué es lo que realmente buscan.

Una fuerte tendencia, complementaria de la anterior, es la de buscar la cosmovisión de los grupos étnicos actuales sobre todo en los "restos" precolombinos, excluyendo o secundarizando los contenidos generados por el catolicismo desde finales del siglo XV, pese a que tienen una fuerte presencia en las comunidades étnicas y no étnicas, comenzando con los usos del alcohol en toda una serie de rituales de sanación, y también en rituales no referidos a procesos de s/e/a-p, pero que son parte de la estructura e identidad de estos grupos. Pero, además, Ilama la atención que se suele consignar más la presencia de saberes espiritistas o de ciertas religiosidades recientes, que las que han sido generadas a partir del catolicismo.

Considero que la concepción del mundo y, sobre todo, el sentido común -como diría Gramsci- de todos los sectores sociales y, especialmente, de los sectores subalternos, hay que buscarlos en la vida cotidiana de los sujetos y grupos para ver lo que los sujetos hacen/viven con ellos. Y así observamos, que un gran número de estudios antropológicos consideran que las cosmovisiones precolombinas fundamentan los saberes de los grupos étnicos actuales sobre las enfermedades 
que padecen, en términos de una armonía y equilibrio entre la comunidad, el sujeto y "la naturaleza". Lo cual puede ser, pero ocurre que, cuando estos autores presentan sus etnografías, encontramos que la desarmonía y el desequilibrio no son referidos por los sujetos y microgrupos a las cosmovisiones, sino a las envidias suscitadas entre sujetos, microgrupos e inclusive comunidades, y organizadas frecuentemente en torno a procesos económicos y simbólicos, como la Antropología lo viene demostrando desde por lo menos la década de 1930, y que explicaba a través de las teorías de Foster ${ }^{(27)}$, Erasmus ${ }^{(28,29)}$ o De Martino ${ }^{(30)}$. Y digo explicaba, porque actualmente no tenemos nuevas teorías que nos expliquen antropológicamente el papel de la envidia en el caso de los pueblos originarios y no originarios.

La revisión bibliográfica indica que la mayoría de los episodios de enfermedad fueron referidos por los entrevistados a las relaciones conflictivas que han tenido con sujetos/microgrupos, a transgresiones a las normas comunitarias, al papel de los brujos y/o a los designios divinos o mágicos. La mayoría de los sujetos entrevistados no atribuye sus enfermedades a la pérdida de las relaciones armónicas naturaleza/sociedad/sujeto ni al desequilibrio cósmico, sino a la relación frío/caliente cotidiana, a lo que le pasó con un vecino, a que no satisfizo un antojo, a que no tiene dinero y/o leche para alimentar a su hijo recién nacido, a que vio determinadas escenas sexuales que no debería ver a su edad o a que dios lo envió o lo quiso así.

Los sujetos utilizan también explicaciones físicas y orgánicas de tipo tradicional o alopática, e incluso el uso simultáneo o secuencial de varias de las explicaciones señaladas. Y en todas ellas puede operar el poder de los brujos o los designios mágicos o divinos, subrayando que reiteradamente se verifica la propuesta de Evans-Pritchard( ${ }^{(31)}$ respecto del uso simultáneo de diagnósticos y terapéuticas "empíricas" y "mágicas" por parte del paciente y su familia en grupos africanos. Y más allá de las críticas formuladas a dicha propuesta, esto no nos debe llevar a ignorar la cosmovisión, sino a ver si es parte del sentido común y de las prácticas que los sujetos y grupos de los diferentes sectores sociales generan y utilizan actualmente en su vida cotidiana respecto de los procesos de s/e/a-p.

Tenemos que dejar de buscar "a como sea" las cosmovisiones ancestrales en los actores sociales actuales, si es que queremos comprenderlos a ellos, pues si no lo hacemos, no vamos a entender, por ejemplo, por qué los jóvenes actuales ya no quieren ser chamanes o brujos, ni tampoco parteras "empíricas", aunque algunos quieren ser auxiliares de salud, promotores, enfermeras y médicos. $\mathrm{Ni}$ vamos a comprender tampoco por qué padres indígenas, sobre todo en medios urbanos, dejan de hablarles a sus hijos en su lengua, y no están de acuerdo con una educación bilingüe $o$, aun estando de acuerdo, prefieren que sus hijos aprendan español o inglés. La búsqueda de por lo menos ciertos pasados en los sujetos actuales, conduce casi inexorablemente a encontrar dichos pasados, pero tergiversando los objetivos y orientaciones que una parte de los sujetos y microgrupos operan en su vida cotidiana.

Lo cual no niega -y lo subrayo- que en función de objetivos propios del investigador se busquen las antiguas cosmovisiones y modos de vida para documentar la existencia de otras formas de vivir la realidad, incluidos los procesos de s/e/a-p. Pero asumiendo que gran parte de lo que se llama cosmovisión constituye reconstrucciones intelectuales que se caracterizan por su coherencia y racionalidad, y que tiene poco que ver con la vida cotidiana de las personas caracterizadas por transgresiones, conflictos e incongruencias entre lo que se dice y lo que se hace, o inclusive entre lo que el sujeto dice sobre un mismo problema al tratarlo en diferentes situaciones, lo cual implicaría la necesidad de desarrollar una antropología situacional.

Dichas cosmovisiones suelen ser tipos ideales, que sus constructores $-y$ sobre todo sus seguidores- identifican con la realidad, orientando la mirada de los antropólogos hacia esos "tipos ideales", cuando es en la vida cotidiana donde necesitamos observar lo que los sujetos y microgrupos hacen, piensan, usan, y por supuesto dicen, y no solo a través 
de su propia experiencia, sino de la experiencia con los otros con quienes se relacionan los sujetos. Aclarando que no cuestiono el uso de tipos ideales -o de modelos-, sino que cuestiono identificarlos con la realidad.

Por lo cual, si bien hay una tendencia creciente a modificar estas orientaciones, todavía domina en muchos autores un mayor interés por las cosmovisiones pasadas en función de evidenciar la diferencia, la legitimidad, las capacidades de sociedades que fueron subalternizadas y explotadas. Y que ha conducido, por ejemplo, a que una parte de los que estudian procesos de s/e/a-p les preocupe mucho más la muerte que la mortalidad. De tal manera que ante grupos que tuvieron y tienen altísimas tasas de mortalidad infantil, les interesa más los rituales de sanación o de muerte a través de los cuales constatar la presencia de cosmovisiones, que analizar en términos etnoepidemiológicos las mortalidades actuales $y$, donde se pueda, también las pasadas. Obviamente, lo que tendríamos que estudiar son tanto los rituales de la muerte como las tasas de mortalidad y los saberes para limitar o impedir la muerte como parte de una epidemiología sociocultural de la mortalidad, pero lo que domina es la polarización de objetivos.

Si bien las tendencias antropológicas más recientes relacionadas con la salud colectiva incorporan cada vez más datos epidemiológicos, necesitamos asumir que la omisión de dichos datos es parte de una tradición antropológica fuerte, ya que tanto en el pasado como en la actualidad los estudiosos de las denominadas enfermedades tradicionales no producen ni usan datos de mortalidad para casi ninguna enfermedad tradicional, salvo en determinados casos relacionados frecuentemente con brujería, pero de los cuales solo nos dicen que pueden ser mortales. Más aún, los estudios sobre curadores tradicionales, incluidas las parteras, se caracterizan porque a ningún curador se le mueren los pacientes, generando la imagen de que todas las muertes son hogareñas u hospitalarias. Es importante señalar que un equipo coordinado por Zolla ${ }^{(32)}$ codificó, para todos los grupos étnicos mexicanos, las principales enfermedades que padecen, enumerando las que pueden causar la muerte. Dicho trabajo constituye un aporte inestimable, pero que no presenta descripciones etnográficas, y menos datos estadísticos respecto de las mortalidades.

Hay además una fuerte tendencia a seguir trabajando exclusivamente con un actor social, en lugar de trabajar con el conjunto de actores significativos que tienen que ver con los procesos de s/e/a-p estudiados. Es decir, todo el peso se pone en el punto de vista de un actor, de tal manera que se puede llegar a obtener una información estratégica y de calidad, pero que no puede explicar determinados procesos. Y más aún, puede deformar la interpretación de los procesos de s/e/a-p estudiados. Como vengo señalando desde hace demasiados años, si bien reconozco el objetivo ideológico -y por supuesto metodológico- de esta manera de trabajar, creo que limita el conocimiento del problema, y a la larga también limita el cumplimiento de los objetivos ideológicos. Por lo cual consideramos necesario impulsar el desarrollo de un enfoque relacional.

En los últimos años, han surgido toda una serie de estudios sobre las narrativas de los procesos de s/e/a-p que, más allá de sus logros testimoniales, no han reflexionado demasiado sobre las críticas que se han hecho a estas corrientes en los países donde se desarrollaron desde, por lo menos, la década de 1970, y en los cuales no solo se cuestiona la literaturización de los padecimientos, sino la eliminación de lo cultural y de lo económicopolítico quedando reducida la enfermedad a la subjetividad.

Un sujeto del cual desaparecen además las rutinas y las repeticiones que caracterizan toda vida, y donde los usos y costumbres pueden aparecer como productos individuales y frecuentemente heroicos ${ }^{(33,34)}$. De tal manera que hemos ido pasando de una antropología de "la" cultura, de las normas, de los roles, es decir, de la rutina y repetición, a una antropología del acto o, por lo menos, narración individual. La antropología social, la sociología y, en cierta medida, la psicología social se constituyeron a través de observar la vida como rutina y repetición, lo cual secundarizaba el 
cambio y, sobre todo, al sujeto; pero actualmente hemos pasado a una antropología que ignora o secundariza las pautas, las normas o lo práctico-inerte como llamaba Sartre a la rutina y a la repetición.

En América Latina es creciente el número de antropólogos que dicen aplicar un enfoque fenomenológico lo cual, especialmente en Brasil, tiene un desarrollo de casi tres décadas, a través de autores que lo han aplicado con idoneidad ${ }^{(35,36)}$. En forma explícita o tácita, una parte de estos estudios ha cuestionado los trabajos de orientación cognitiva, semiótica y hermenéutica, por haber reducido los procesos de s/e/a-p -y especialmente el cuerpo- a representaciones sociales, a textos y a interpretaciones, donde no solo desaparecen las experiencias sino también se reduce o elimina el papel de las prácticas sociales.

Sin embargo, pese a estos aportes, tengo serias dudas sobre la fenomenología que están utilizando muchos de los que aplican este enfoque. Cuando les he preguntado a varios autores ¿qué tipo de fenomenología aplican?, casi siempre comienzan hablando de Husserl y, sobre todo, de Merleau Ponty y la corporización, pero sin precisar lo que hacen. Como sabemos, tanto Husserl ${ }^{(37)}$ como estudiosos de la religión como Leeuw ${ }^{(38)}$ plantean como decisivo aplicar la reducción fenomenológica, ya que:

\section{...la investigación de la conciencia implica que hay que dejar de lado todas las teorías sobre la conciencia, todas las opiniones preconcebidas y explicacio- nes, para observar con la mayor ausencia de prejuicios lo que sucede en mi con- ciencia aquí y ahora. ${ }^{(39)}$}

Más aún, para Safranski "La reducción fenomenológica es el aspecto de la fenomenología que lo decide todo"(39). Y si bien otras tendencias fenomenológicas no aplican esta reducción, no obstante señalan cómo hacen para ir "hacia las cosas mismas"; lo cual no observo en los trabajos publicados por antropólogos latinoamericanos, sino excepcionalmente. Más aún, a partir de sus textos no alcanzo a diferenciar lo que sería un informe fenomenológico de lo que sería un informe etnográfico. Tengo la impresión de que lo que hacen es una de las tantas variantes de la etnografía, rescatando algunos aspectos individuales de los actores sociales y referidas a temáticas poco tratadas hasta hace pocos años, como es el caso de las referidas al "cuerpo".

$Y$ esta ausencia de claridad y reflexividad antropológica Ilama la atención sobre todo porque varios antropólogos no solo hacen referencia a la fenomenología, sino que simultáneamente adhieren a las propuestas de Bourdieu. Pero ocurre que para este autor y sus discípulos ${ }^{(40,41)}$, el punto de partida metodológico debe ser la puesta en evidencia de los presupuestos/prejuicios del investigador, por varios motivos $y$, en especial, para que los estudios no constituyan -como señaló reiteradamente Bourdieu- "profecías autocumplidas". Asumiendo además que, en el caso de la antropología social latinoamericana, hay un notorio peso de los presupuestos y objetivos ideológicos de muy diverso tipo, lo cual he señalado reiteradamente en varios de mis textos, y que no cuestiono, pues es parte "de nuestra manera de estar en el mundo".

Pero lo que no observo, tanto en términos fenomenológicos como de Bourdieu, es una reflexión sobre el papel que tienen dichos presupuestos, ni cómo son manejados metodológicamente, o cómo inciden en la etnografía y en las explicaciones producidas. Más aún, domina cierta tendencia a mezclar teorías y conceptos contradictorios; y así, en el caso de Bourdieu observamos que pese a que su propuesta teórica sobre el habitus y el campo exige que se incluya la posición de los diferentes actores sociales que compiten por objetivos específicos, ocurre sin embargo que la casi totalidad de los que la usan centran su etnografía y análisis en un solo actor social.

Hay un aspecto metodológico que domina no solo la producción en antropología médica, sino también en antropología social y en etnología, y cuya importancia radica en que constituye la "técnica" con que estereotipadamente los antropólogos nos identificamos $y$, en gran medida, nos diferenciamos: 
me refiero a la denominada observación participante, que la inmensa mayoría de los antropólogos dicen que practican, pero que la mayoría -incluyéndome- no realizamos o realizamos parcialmente; porque la observación participante implica observación y los antropólogos no aprendemos a observar, ni hacemos observación sistemática, y por ello nuestros trabajos se basan casi exclusivamente en la palabra del otro.

Desde, por lo menos la década de 1980, antropólogos de EEUU y de países europeos $^{(42,43)}$ no solo están preocupados por la medicalización, sino por el peligro de medicalización de la antropología médica ${ }^{(44,45,46)}$, lo cual también se está dando en América Latina $^{(47)}$. Gran parte -aunque no exclusivamente- de la medicalización se observa en los proyectos antropológicos caracterizados por la intervención, de tal manera que varios de los estudios sobre salud reproductiva y sobre interculturalidad utilizan enfoques más biomédicos que antropológicos, más allá de que apelen a conceptos como cultura y subjetividad. Y ello, a veces, es debido a que, por lo menos una parte de las intervenciones sobre los procesos de s/e/a-p simplifican, esquematizan y excluyen aspectos de la realidad para posibilitar la intervención, sobre todo, cuando pretende ser generalizada. Por lo cual procesos complejos son esquematizados, simplificados o excluidos para poder intervenir; de tal manera que, por ejemplo, las actividades de interculturalidad en México -aún programadas por antropólogos- decidieron excluir a los chamanes y brujos de sus propuestas de intervención, porque el sector salud se oponía, aunque permitió el trabajo de parteras y herbolarios.

\section{APORTES Y PROPUESTAS}

Según Maria Cecília Minayo ${ }^{(48)}$, la antropología médica es actualmente una de las disciplinas más dinámicas de las que componen el campo de la salud colectiva en Brasil, no solo por su notable producción bibliográfica, sino por sus contribuciones al pensamiento de la salud colectiva. Y subrayando que la antropología médica no constituye una disciplina subordinada a la medicina, sino una disciplina que desarrolla sus propias perspectivas y que genera aportes teóricos y metodológicos. A su vez, Sebastián García, Director de Calidad y Educación para la Salud de la Secretaría de Salud de México, señaló que las nuevas áreas que se requieren para fortalecer las distintas ramas de la medicina son física médica, ingeniería médica, robótica y antropología de la salud ${ }^{(49)}$. Es decir, el reconocimiento de la antropología médica latinoamericana viene tanto desde la propia disciplina, como desde la biomedicina.

La antropología médica latinoamericana ha realizado notorios aportes en muy diferentes campos y problemáticas, y así ha fundamentado e impulsado el uso de técnicas y metodologías cualitativas en el estudio de los procesos de s/e/a-p, obteniendo información e interpretaciones estratégicas que no se logran a través de técnicas y aproximaciones estadísticas, lo cual ha conducido a un uso intensivo de estas técnicas por parte de investigadores biomédicos, cuyos usos han sido frecuentemente cuestionados por los antropólogos.

La antropología médica no solo ha evidenciado la articulación que los sujetos y grupos hacen entre las diferentes formas de atención que operan en un contexto determinado, sino el proceso de apropiación que todos los sectores sociales hacen de los saberes biomédicos. Ha señalado que las medicinas tradicionales y alternativas forman parte normalizada de las trayectorias de atención de la enfermedad, y que todas las formas de atención -y no solo la biomedicina- se caracterizan por el cambio. Ha demostrado que la sociedad y la cultura, así como también la biomedicina y los curadores tradicionales pueden producir enfermedades. Ha incluido cada vez más los procesos de poder en toda relación curador/paciente, así como en otras relaciones, en especial, en las de género. Ha subrayado permanentemente la importancia del trabajo de campo frente al desarrollo constante e intermitente de propuestas "teoricistas". Ha seguido planteando dentro de lo 
posible, una visión holística de los procesos de s/e/a-p que cuestiona la orientación unilateral y excluyente del modelo biomédico.

Uno de sus aportes más constantes es el de documentar y tratar de entender los saberes populares respecto de los procesos de s/e/a-p; todas las corrientes, aun las más antagónicas, han tratado de describir la racionalidad de los diferentes grupos -especialmente de los étnicos- para explicar y actuar contra los padecimientos que los afectan, así como para entender sus comportamientos de rechazo supuesto o real hacia la biomedicina, pero subrayando que a diferencia de lo sostenido por autores como Byron Good ${ }^{(50)}$ respecto de los antropólogos -supongo- de su país, gran parte de los antropólogos latinoamericanos ha tratado de describir y entender los saberes subalternos sin considerarlos falsos o equivocados, más allá de la eficacia que pueden tener, lo cual constituye uno de los puntos de mayor distanciamiento con los saberes e instituciones biomédicos.

Frente a las preguntas médicas de por qué los hipertensos, los diabéticos o los sujetos con VIH-sida no cumplen con los tratamientos "indicados"; o por qué se ha desarrollado un rechazo creciente a las diferentes vacunas, los antropólogos tratan de encontrar la racionalidad no solo cultural, sino también económica, política e ideológica de esos comportamientos referidos además a los contextos dentro de los cuales desarrollan dichos comportamientos. $Y$ buscan una racionalidad que parte del supuesto de que la evidencia científica, por lo menos de manera inmediata, no cambia los comportamientos de determinados sectores sociales. Y más aún, en determinados sectores sociales se utilizan argumentos científicos y nuevos medios tecnológicos para justificar, por ejemplo, el rechazo a las vacunas ${ }^{(51)}$. Lo cual en ciertos casos se refuerza paradojalmente, dado que es el personal de salud quien rechaza vacunarse, como ocurrió durante la epidemia de influenza $A$ (H1N1) en México y en varios países europeos.

Como ya señalé, algunos de los principales aportes antropológicos han sido sus críticas a la biomedicina, incluidas propuestas de cambio. Las críticas se han hecho respecto de aspectos teóricos, metodológicos, de aprendizaje y de intervención y, en particular, de sus formas de actuar con los pueblos "originarios", y últimamente con las mujeres de los diferentes sectores sociales y no solo de dichos pueblos. Estas críticas van desde establecer la imposibilidad estructural de la biomedicina $-y$ especialmente del Sector Salud- de tomar en cuenta y aplicar determinados aspectos culturales, políticos y subjetivos que inciden en los padeceres y su comprensión y solución; hasta los que cuestionan aspectos específicos del saber y las instituciones médicas, aunque confiando en su modificación y complementariedad con las propuestas socioantropológicas.

Mientras las primeras propuestas concluían que hay una incompatibilidad radical entre los enfoques antropológicos y biomédicos, sosteniendo que todo profesional -incluidos los antropólogos- que trabaje dentro de las instituciones biomédicas va a medicalizar no solo sus intervenciones, sino su forma de pensar, lo cual es sostenido sobre todo por autores brasileños ${ }^{(8)}$. Otras tienen expectativas de que puedan mantenerse las formas de pensar y actuar no biomédicas aun trabajando en instituciones biomédicas; a través de una suerte de división del trabajo, en la que la antropología médica se ocuparía básicamente de complementar desde sus perspectivas lo que no desarrolla la biomedicina, ya sea en la formación, en la investigación e incluso en la intervención, especialmente, a través de ONG.

No obstante, todas las tendencias coinciden en que las orientaciones biomédicas dominantes tienden a imponer sus formas de pensar y actuar respecto, por ejemplo, del manejo que hace el sector salud de los grupos de autoayuda; de la manera negativa de pensar la prevención utilizada por los grupos sociales; de las concepciones simplistas que tienen para incidir en los estilos de vida de los conjuntos sociales; del manejo que suelen hacer de las técnicas cualitativas convirtiéndolas en técnicas de investigación rápida o en la estigmatización de la autoatención y especialmente de la automedicación. 
Para los antropólogos latinoamericanos no cabe duda de que los procesos de s/e/ap son parte intrínseca de la cultura dentro de la cual operan; son parte de la identidad cultural, por lo que una parte de estos profesionales ven la expansión de la biomedicina, inclusive en los curadores tradicionales, como una de las principales amenazas no solo respecto de la medicina tradicional sino de la identidad cultural de los grupos étnicos.

Estas maneras antropológicas de entender la biomedicina tendrían que asumir que existen diferencias radicales entre la formación médica y la formación antropológica y, especialmente, en los objetivos profesionales y personales que tienen médicos y antropólogos. Mientras el eje de la formación biomédica es biológica y biologicista, el de los antropólogos ha sido la cultura, los saberes y los significados; y mientras el objetivo de la medicina ha sido intervenir sobre la enfermedad, el de los antropólogos ha sido "comprender". Y por eso y por otras razones, considero necesario que los antropólogos traten de describir y entender la racionalidad biomédica, tal como han venido haciendo respecto de la racionalidad de los saberes populares.

Ahora bien, gran parte de las críticas señaladas refieren a la clínica biomédica, y menos a determinadas corrientes de la medicina social y de la salud pública, con las cuales se coincide en críticas que se realizan hacia las instituciones biomédicas, y también hacia las políticas públicas. Más aún, en ciertos momentos -especialmente en el campo de la salud mental- ha habido coincidencias entre las propuestas antropológicas y las corrientes psicoterapéuticas críticas, como ocurrió durante un tiempo con el proceso de clausura o reformulación de las instituciones manicomiales y la aplicación de determinadas formas de atención y convivencia con la enfermedad mental.

Los antropólogos latinoamericanos han estudiado los saberes que los grupos y las comunidades tienen sobre muy diferentes aspectos de la realidad; aunque estos estudios hasta por lo menos la década de 1990 ignoraban la subjetividad de los actores sociales que estudiaban, pese a que entre 1930 y 1950, se desarrollaron en EEUU toda una serie de corrientes antropológicas que recuperaban al sujeto o por lo menos a la persona. Pero, pese a la influencia señalada de la antropología estadounidense, especialmente, en México y América Central, dichas tendencias fueron frecuentemente cuestionadas por considerarlas "psicologistas" por autores que adherían a propuestas anticolonialistas y/o clasistas.

Pero durante el dominio neoliberal, junto con la diferencia cultural, el empoderamiento y el etnicismo, se recupera la subjetividad; por lo cual, debido sobre todo a la influencia de tendencias desarrolladas en EEUU, se fue generando en la región una antropología que comenzó a tomar en cuenta las aflicciones, los sufrimientos, las experiencias y las emociones, lo que nos parece necesario no solo para incluir la subjetividad, sino para complementar los enfoques previos, y para profundizar la posibilidad de entender aún más las racionalidades culturales a nivel de los grupos y de los sujetos.

Lo interesante es que las tendencias antropológicas que actualmente recuperan al sujeto en la región no suelen hacer referencias, por ejemplo, a la gran cantidad de biografías, autobiografías o historias de vida que desarrollaron antropólogos y sociólogos estadounidenses entre 1930 y 1950. Más aún, en México ya casi no se hacen alusiones a la biografía de Juan Chamula desarrollada por Ricardo Pozas, ni prácticamente a toda la obra de Oscar Lewis, por lo cual considero que, en la antropología social actual, y no solo en América Latina, domina una a-historicidad teórica cada vez más notoria, articulada con ciertos no saberes.

Y así, por ejemplo, pese a que esta es actualmente una de las tendencias más dinámicas, no termino de entender por qué gran parte de los antropólogos que trabajan con el sujeto apelan a las propuestas de Bourdieu y sobre todo de Foucault que excluyen al sujeto, en lugar de utilizar conceptos y propuestas como, por ejemplo, las de Linton $^{(52)}$ o de Sartre $^{(53)}$ que se caracterizan justamente por incluir al sujeto, por supuesto que con otros nombres. Posiblemente, esta incongruencia 
explica por qué la mayoría de los que utilizan conceptos como sujeto, subjetividad y experiencia no los definen.

Linton desarrolló en la década de 1940 un modelo conceptual mucho más dinámico que el estructuralista de Bourdieu, al proponer que si queremos entender lo que Bourdieu Ilamó habitus, tendríamos que estudiar simultáneamente las pautas ideales, las reales y las construidas que utilizan los grupos y sujetos. Por supuesto que lo señalado no cuestiona a Bourdieu respecto de lo que a él le interesa estudiar, sino que plantea que para los antropólogos que quieren trabajar con el sujeto, es mucho más pertinente hacerlo con la propuesta de Linton ${ }^{(52)}$ o con la del Sartre de Cuestiones de método ${ }^{(53)}$. Pero las modas son las modas, y los "olvidos" también.

Considero que uno de los principales aportes de la antropología médica es evidenciar que gran parte de las enfermedades tradicionales han sido generadas por relaciones sociales, de tal manera que, para los sujetos, microgrupos y comunidades, las causales de los padecimientos físicos, mentales o psicosomáticos son los conflictos que operan a nivel familiar o entre vecinos. $Y$ este es uno de los aspectos que más deberíamos estudiar, y no solo respecto de las enfermedades tradicionales; dado que explícitamente o no, los enfoques antropológicos han considerado que las enfermedades tradicionales y no tradicionales tienen componentes socioculturales y psicosomáticos, en las que operan los significados y acciones de los sujetos y microgrupos.

A partir del material sobre enfermedades tradicionales compilado por Zolla ${ }^{(33)}$ y colaboradores para México, he detectado alrededor de cincuenta padecimientos tradicionales que son generados por relaciones sociales, de los cuales varios constituyen los padecimientos más frecuentes que sufren los sujetos de los grupos étnicos mexicanos, siendo varios de ellos de tipo mortal. Si bien muchos de estos padecimientos son atribuidos a Dios o a personajes míticos, la descripción etnográfica evidencia que no solo gran parte de los padecimientos son generados por conflictos o problemas sociales cotidianos que tienen que ver con la propiedad de la tierra, con los convenios matrimoniales entre familias o con violencias de diferentes tipos; sino que, como señalé previamente, en la mayoría de estas relaciones interviene la envidia y remite a situaciones de pobreza, lo cual ha sido descripto e interpretado desde la década de 1940 por culturalistas, funcionalistas, estructuralistas y marxistas.

\section{DUDAS E INCERTIDUMBRES}

Ahora bien, respecto de varios de los procesos y problemas que estudia la antropología médica en América Latina, emergen dudas sobre algunos de los objetivos y consecuencias en las cuales participó de diferentes formas nuestra disciplina, y más allá de las formas en que participó. Y así, como sabemos, en varios países de la región se crearon durante este lapso servicios especiales de salud para los grupos étnicos, y se aplicaron políticas interculturales de salud apoyadas inclusive por la Organización Panamericana de la Salud. Aunque subrayando que la mayoría de estos servicios carecen de financiamiento o es mínimo; y que las políticas de salud intercultural tuvieron un escaso impacto y se caracterizan -salvo excepciones- por su discontinuidad o por su fracaso como es, por ejemplo, el caso de los hospitales mixtos. Recordando que varias de las tendencias interculturales que se desarroIlaron desde la década de 1980 proponían y esperaban una suerte de articulación entre la biomedicina y la medicina tradicional que no se cumplió, lo cual verificó para muchos antropólogos la incompatibilidad que existe entre antropología y biomedicina. Pero lo que considero relevante es analizar no solo si es viable un sistema de salud especial para los pueblos originarios, sino lo que ello supone en términos económicos, políticos, biomédicos y, también, raciales.

Uno de los señalamientos explícitos o tácitos de los estudios antropológicos refiere a la paulatina o rápida expansión de la biomedicina sobre los pueblos originarios, 
articulada de diferentes maneras con los saberes locales, pero con cada vez más hegemonía, debido a varios procesos conocidos por todos. La mayoría de los señalamientos antropológicos hacia dicha expansión son críticos, pero estos ¿limitan, impiden, modifican o no dicha expansión? Pero, además, ¿qué balance en términos puntuales hacemos de dicha expansión biomédica?, ¿ha sido positiva, negativa o ambivalente y respecto de qué problemas?

Si bien no solo en las propuestas antropológicas, sino en las de los aparatos médico/ sanitarios y de la Organización Panamericana de la Salud (OPS), se especifica la necesidad de mejorar la relación médico/paciente y de impulsar la comprensión del personal de salud respecto de las características socioculturales de los pacientes y no solo de los pertenecientes a los grupos étnicos; vemos sin embargo que estos objetivos pasan a ser secundarios frente a situaciones más decisivas, como son las referidas a la persistencia de las desigualdades en atención de la salud y las escasas y diferenciales inversiones en salud en general y especialmente en la de los grupos indígenas por parte de los gobiernos federales y locales. Lo cual se articula con la creciente demanda de servicios de salud por los sectores sociales subalternos, y de los tiempos cada vez más reducidos de la consulta médica. Si bien ambos objetivos no son antagónicos, la cuestión está ien dónde colocamos el peso del desarrollo de los sistemas de salud en términos "ideales" y en términos de posibilidades?

Lo cual se relaciona con un proceso que me ha preocupado reiteradamente y que tiene que ver, en términos simplificados, con la capacidad de agencia de los sectores subalternos respecto de los procesos de s/e/a$\mathrm{p}^{(54) ;}$ con la posibilidad de generar organizaciones autónomas y alternativas por parte de la sociedad civil que no sean cooptadas o reorientadas por las fuerzas sociales dominantes; así como de generar resistencias, empoderamientos o acciones concretas alternativas respecto de los procesos de s/e/a-p. Y lo que observo me preocupa, ya que de mi revisión surge que los procesos de s/e/a-p no aparecen como centrales para los movimientos étnicos ni para sus líderes más que en términos retóricos. Es decir, salvo excepciones, no localizamos movimientos que realmente impulsen las formas de sanación propias como alternativas posibles a las biomédicas. Lo que sí registramos es el impulso de pequeños grupos de activistas en el caso del VIH-sida, y especialmente en el caso de las mujeres, pero centradas casi exclusivamente en las violencias y en los procesos de salud reproductiva. Recordando que en estos grupos encontramos diversos tipos de profesionales, incluidos antropólogos.

Pero además, salvo en el caso de los grupos tipo Alcohólicos Anónimos caracterizados por su autonomía, ¿cuáles son los grupos, asociaciones o movimientos referidos a procesos de s/e/a-p que han sido creados, organizados, mantenidos, financiados por sectores sociales subalternos, ya sea para demandar mejores servicios biomédicos, o para impulsar formas tradicionales o alternativas de sanación? Los que conozco $-y$ solo me refiero a México- han sido creados y organizados por sujetos y grupos pertenecientes a sectores sociales medios, incluidos intelectuales y profesionales que han liderado dichos grupos. Y al señalar esto, no niego que luego de este "disparador" organizador, sujetos y microgrupos impulsen dichos procesos, aun cuando estos siguen siendo financiados externamente. Pero además me preocupa la penetración de la industria químico/farmacéutica en el financiamiento y liderazgo de estos grupos, por lo menos en países europeos y en EEUU, ya que no contamos con estudios para América Latina.

Por otra parte, especificar qué es resistencia, no solo en términos de movimientos sociales sino individuales, microgrupales o comunitarios, implica decisiones interpretativas difíciles de establecer. ¿Cuándo la no ida al médico es producto de la falta de médicos o de recursos económicos, y cuándo es una actitud negativa hacia la biomedicina? ¿Cuándo la automedicación constituye una forma de empoderamiento respecto de la biomedicina, y cuándo un proceso social que racionaliza el tiempo propio o determina 
lo que es más económico para el sujeto/ microgrupo? (55,56) $^{2}$.

Pero lo central para nosotros es poder establecer la capacidad de agencia de los sujetos y grupos subalternos no solo para autoatenderse o resistir, sino para negociar o incluso imponer propuestas para mejorar o modificar las actividades y políticas de salud que operan en sus territorios. Y este es uno de los procesos que más deberíamos analizar en cada uno de los países latinoamericanos.

Ahora bien, como ya señalé, la bibliografía ha documentado que gran parte de las enfermedades tradicionales, expresan conflictos sociales generalmente entre personas cercanas que operan dentro de contextos de pobreza y de escasez. Pero según varios autores, una parte de las enfermedades tradicionales expresan también las limitaciones o impedimentos que tienen ciertos actores sociales especialmente niños, jóvenes y sobre todo mujeres en el desarrollo de determinados comportamientos o si se prefiere "deseos". Lo cual, por ejemplo, puede conducir a que para una mujer en determinadas comunidades, una de las posibles conductas que tiene al negarse a contraer matrimonio con la persona elegida/convenida por su familia, es contraer un padecimiento que es reconocido por la comunidad y que puede ser atendido por un curador tradicional(57).

Lo cual implica que los denominados "usos y costumbres" pueden generar consecuencias negativas para algunos de los miembros de las comunidades, que a veces son "solucionadas" a través de enfermedades u otros mecanismos, pero frecuentemente no. Pese a ello, las diversas corrientes antropológicas y no solo las culturalistas e indianistas, han reconocido de modo totalizante el papel positivo y la legitimidad de los usos y costumbres, lo cual ha sido reforzado durante el lapso que estamos analizando por el desarrollo de los movimientos étnicos y por el empoderamiento político de algunos de esos grupos.

Recordemos que, mientras una parte de estos movimientos y analistas niega -o por lo menos no menciona- las posibles consecuencias negativas de determinados usos y costumbres, ya que solo suelen identificarlos con el denominado "buen vivir" o con el "comunalismo"; otras tendencias, cuya principal expresión ha sido la antropología feminista, señalan que determinados usos y costumbres limitan, anulan, excluyen el papel de la mujer, sometiéndola a condiciones de inferioridad que justifican culturalmente -entre otras acciones- la aplicación de violencias de diferente tipo, que pueden concluir en la muerte.

Esta tendencia crítica se afirma además a través de estudios sobre procesos de s/e/a-p que evidencian la situación actual de ciertos actores sociales, a nivel general, y de los grupos étnicos, en particular, respecto de ciertos padecimientos. Y así, es cada vez más frecuente encontrar propuestas que ante la gravedad de ciertos problemas, como, por ejemplo, la expansión del VIH-sida en pueblos indígenas y no indígenas de zonas rurales, plantean que:

...los antropólogos no podemos seguir reproduciendo la defensa a ultranza de los usos y costumbres como status quo y factores de preservación cultural. Debemos aportar los elementos necesarios para que se adopte un concepto dinámico, en el que los pueblos indígenas tengan todo el derecho de transformar su propia cultura y a sí mismos. Igualmente, nos corresponde el papel de proveer los insumos teóricos y analíticos para entender la vulnerabilidad y las mejores maneras de luchar contra ella [...] eso implica muchos retos, tal vez el más importante e imprescindible sea que las comunidades asuman la responsabilidad de hablar de sexualidad, y en particular de diversidad amorosa/sexual. ${ }^{(58)}$

Obviamente, lo que proponen estos y otros autores ${ }^{(59,60,61)}$ no refiere solo a los usos y costumbres de los pueblos originarios, sino a todos los que en diferentes sociedades favorecen el desarrollo de padecimientos, incluso letales, especialmente en algunos actores sociales confinados a determinados roles y estatus.

Los usos y costumbres refieren a la comunidad, pero a una comunidad donde ciertos actores sociales pueden ejercerlos sin 
problemas, mientras que para otros están restringidos. Dentro de las comunidades, por más homogéneas que sean, siempre existen actores con mayores posibilidades y beneficios diferenciales, y no solo en términos socioeconómicos, sino también de poder y culturales.

Pero hay que señalar que si bien el texto de Ponce y Nuñez propone acciones concretas, sin embargo, no aclara un problema básico, ya que no indica qué podemos o debemos hacer ante los grupos originarios y no originarios que no quieren modificar su propia cultura respecto de enfermedades que los están afectando y hasta diezmando, prefiriendo seguir con sus usos y costumbres, como ocurrió recientemente en varios países africanos con la enfermedad de Ébola o en el cordón calvinista holandés respecto de la epidemia de influenza $A(\mathrm{H} 1 \mathrm{~N} 1)$, ya que este no es solo un problema latinoamericano ni exclusivamente étnico.

Necesitamos asumir que para los antropólogos médicos latinoamericanos los procesos de s/e/a-p son parte intrínseca de la cultura de todo grupo social, y especialmente de los pueblos originarios, por lo que ven la expansión de la biomedicina como una de las principales amenazas a la identidad cultural de dichos pueblos. Y esta expansión suele ser analizada en términos del dominio creciente de una racionalidad instrumental y de una hegemonía biomédica, que puede ser cuestionada apelando a las medicinas nativas. $Y$ si bien la teorización de estas propuestas no suele ser realizada por antropólogos médicos latinoamericanos, una parte de ellos adhiere a los que nos hablan de las epistemologías del sur o a las orientaciones autodenominadas decoloniales. Pero ocurre, que estas propuestas no solo son básicamente ideológicas, sino que, en el caso de las primeras, repiten lo que parte del pensamiento europeo desarrolló entre 1920 y 1950; y en el de los decoloniales no van más allá de las propuestas de teorías como las de la "negritud" o de la "decadencia de occidente".

La señalada constituye la última incertidumbre teórica e ideológica que presento en este texto y que, como siempre, se resuelve en la práctica; una práctica que indica que cada vez más los antropólogos optamos por la intervención o, por lo menos, su acompañamiento, solucionando nuestros conflictos y contradicciones apelando a las metodologías situadas, comprometidas o simplemente útiles.

\section{REFERENCIAS BIBLIOGRÁFICAS}

1. Aguirre Beltrán G. Antropología médica. México DF: CIESAS; 1986.

2. Nigenda G, Duarte-Gomez MB, Navarro RC. L'anthropologie médicale au Mexique: tendances récentes dans la recherche et l'enseignement. En: Saillant F, Genest S, (dirs.). Anthropologie médicale: ancrages locaux, défis globaux. Québec: Les Presses de I'Université Laval; 2005. p. 133-154.
3. Osorio Carranza RM. La antropología médica en México: los caminos recorridos y las nuevas veredas de investigación. AM: Rivista della Società Italiana di Antropologia Medica. 2012;2932:29-58.

4. Vargas L, Casillas LE. Medical anthropology in Mexico. Social Science \& Medicine. 1989;28(12): 1343-1349.

5. Canesqui AM. Os estudos de antropologia da saude/doenca no Brasil na década de 1990. Ciencia \& Saude Coletiva. 2003;8(1):109-124. 
6. Langdon EJ, Folleer M-L. Anthropology of health in Brazil: a border discourse. Medical Anthropology. 2012;31(1):4-28.

7. Minayo MCS. A produção de conhecimentos na interface entre as ciências sociais e humanas e a saúde coletiva. Saúde e Sociedade. 2013;22(1):21-31.

8. Sarti CA. Saude e sofrimento. En: Martins CB, Duarte LF, (coords.). Horizontes das ciências sociais no Brasil: antropologia. São Paulo: ANPOCS; 2010. p. 197-223.

9. Teixeira CC, Garnelo L, (orgs.). Saude indígena em perspectiva: explorando suas matrizes históricas e ideológicas. Rio de Janeiro: Fiocruz; 2014.

10. Garnelo L, Maquiné A. Financiamiento e gestao do subsistema de saude indígena: consideracoes a luz dos marcos normativos da adminstracao pública no Brasil. En: Langdon EJ, Cardoso MD, (orgs.). Saude indígena: políticas comparadas na America Latina. Florianapolis: Editora da UFSC; 2015. p. 107-143.

11. Aguirre Beltrán G. Obra polémica. México DF: Instituto Nacional Indigenista; 1976.

12. Bonfil Batalla G. Diagnóstico sobre el hambre en Sudzal, Yucatán: un ensayo de antropología aplicada. México DF: Instituto Nacional de Antropología e Historia; 1962.

13. Rozitchner L. La izquierda sin sujeto. En: Rozitchner L. Las desventuras del sujeto político: ensayos y errores. Buenos Aires: El cielo por asalto; 1996. p. 45-75.

14. Menéndez EL. Las instituciones y sus críticos o la costumbre de polarizar la realidad: el caso de la influenza A (H1N1). Salud Colectiva. 2014;10(1):15-40.

15. Naciones Unidas, Comisión Económica para América Latina y el Caribe, Organización Panamericana de la Salud. Mortalidad infantil y en la niñez de pueblos indígenas y afroamericanos de América Latina [Internet]. Santiago de Chile: Naciones Unidas; 2009 [citado 01 abr 2018]. Disponible en: https://tinyurl.com/yabxozmu.

16. Coimbra Jr CEA, Santos RV, Escobar AL, (orgs.). Epidemiologia e saude dos povos indígenas no Brasil [Internet]. Rio de Janeiro: Fiocruz, Abrasco; 2005 [citado 01 abr 2018]. Disponible en: https://tinyurl.com/y9wfjvbu.

17. Freire GN, Tillet A, (eds.). Salud indígena en Venezuela. Caracas: Dirección de Salud Indígena; 2007.
18. Instituto Nacional Indigenista. Estado del desarrollo económico y social de los pueblos indígenas de México: primer informe. México DF: Instituto Nacional Indigenista; 2000.

19. Organización Panamericana de la Salud. Una visión de salud intercultural para los pueblos indios de las Américas. Washington DC: Organización Panamericana de la Salud; 2008.

20. Langdon EJ, Cardoso MD, (orgs.). Saude indígena: políticas comparadas na America Latina. Florianópolis: Editora da UFSC; 2015.

21. Arganis Juárez, Elia Nora. Diabetes ayer y hoy: una reflexión de los estudios antropológicos sobre diabetes mellitus. México: CIESAS; 2018.

22. Antonovsky A. The salutogenic model as a theory to guide health promotion. Health Promotion International. 1996;11(1):11-18.

23. Fassin D. Entre las políticas de lo viviente y las políticas de la vida: hacia una antropología de la salud. Revista Colombiana de Antropología. 2004;40:283-318.

24. Imberton Deneke GM. La vergüenza: enfermedad y conflicto en una comunidad chol, San Cristóbal de las Casas. Chiapas: UNAM; 2002.

25. Cuadriello H, Megchún R. El desplazamiento de curanderos y brujos entre los tzeltales de Las Cañadas de la selva lacandona, Chiapas. En: Bartolomé $\mathrm{M}$, Barabas $\mathrm{A}$, (coord.). Los sueños y los días: chamanismo y nahualismo en el México actual. México DF: Instituto Nacional de Antropología e Historia; 2013. p. 213-228.

26. Freire G. Política de salud indígena en Venezuela: con un comentario sobre la participación social y el contexto. En: Langdon EJ, Cardoso MD, (orgs.). Saude indígena: políticas comparadas na América Latina. Florianópolis: Editora da UFSC; 2015. p. 61-82.

27. Foster G. Peasant society and the image of limited good. American Anthropologist. 1965;67(2): 293-315.

28. Erasmus CJ. Man takes control: cultural development and American aid. Minneapolis: University of Minnesota Press; 1961.

29. Erasmus CJ. El síndrome del "encogido" y el desarrollo de la comunidad. América Indígena. 1969;29(1):228-292.

30. De Martino E. II mondo mágico: prolegomini a una storia del magismo. Torino: Einaudi; 1948. 
31. Evans-Pritchard EE. Witchcraft, oracles and magic among the Azande. Oxford: Clarendon Press; 1937.

32. Zolla C, (dir.). Diccionario enciclopédico de la medicina tradicional mexicana. México DF: Instituto Nacional Indigenista; 1994.

33. Bibeau G, Corin E. Culturaliser l'épidémiologie psychiatrique. Les systèmes de signes, de sens et d'action en santé mentale. En: Charest $P$, Trudel $F$, Breton $\mathrm{Y}$, (eds.). Marc-Adélard Tremblay ou la construction de I'anthropologie québécoise. Québec: Presses de l'Université Laval; 1994. p. 105-148.

34. Gledhill J. Power and its disguises: anthropological perspectives and politics. London: Pluto Press; 1999.

35. Rabelo MCM, Alves PCB, Souza IMA. Experiência de doença e narrativa. Rio de Janeiro: Fiocruz; 1999.

36. Maluf SW, Tornquist CS, (orgs.). Gênero, saúde e aflição: abordagens antropológicas. Florianópolis: Letras Contemporâneas; 2010.

37. Husserl E. La crisis de las ciencias europeas y la fenomenología transcendental: una introducción a la filosofía fenomenológica. Barcelona: Crítica; 1991.

38. Leeuw G. Fenomenología de la religión. México DF: Fondo de Cultura Económica; 1975.

39. Safranski R. Un maestro de Alemania: Heidegger y su tiempo. Barcelona: Tusquets; 2010.

40. Bourdieu P, Chamboredon J-C, Passeron J-C. El oficio de sociólogo: presupuestos epistemológicos. México DF: Siglo XXI Editores; 1975.

41. Champagne $P$, Lenoir R, Merllie D. Iniciación a la práctica sociológica. México DF: Siglo XXI Editores; 1993.

42. Conrad P, Schneider JW. Deviance and medicalization: from badness to sickness. St. Louis: Mosby; 1980.

43. Conrad P. Medicalization and social control. Annual Review of Sociology. 1992;18:209-232.

44. Baer HA. The possibilities and dilemmas of building bridges between critical medical anthropology and clinical anthropology: a discussion. Social Science \& Medicine. 1990;30(9):1011-1013.

45. Morgan LM. The medicalization of anthropology: a critical perspective on the criticalclinical debate. Social Science \& Medicine. 1990;30(9):945-950.
46. Singer $M$. The limitations of medical ecology: the concept of adaptation in the context of social stratification and social transformation. Medical Anthropology 1989;10(4):223-234.

47. Menéndez EL. Miradas antropológicas o miradas medicalizadas: Reflexiones sobre las diferencias entre ciencias médicas y antropología social. En: García Acosta V, De la Peña G, (coords.). Miradas concurrentes: La antropología en el diálogo interdisciplinario. México DF: Publicaciones de la Casa Chata; 2013. p. 225-252.

48. Minayo MCS. Contribucoes da antropologia para pensar a saude. En: Campos GWS. Tratado de Saude Coletiva. São Paulo: Hucitec Editora; 2006. p. 189-218.

49. Demandan preparación de especialistas mixtos. Reforma; 24/01/2016.

50. Good BJ. Medicine, rationality and experience: an anthropological perspective. Cambridge: Cambridge University Press; 1994.

51. Hornsey MJ, Harris EA, Fielding KS. The psychological roots of anti-vaccination attitudes: a 24-nation investigation. Health Psychology. 2018;37(4):307-315.

52. Linton R. Cultura y personalidad. México DF: Fondo de Cultura Económica; 1945.

53. Sartre JP. Crítica de la razón dialéctica. Buenos Aires: Editorial Losada; 1968.

54. Berrío Palomo LR. Una década de intervenciones gubernamentales y desde la sociedad civil en torno a la salud materna en Guerrero. En: Freyermuth Enciso MG, (coord.). Salud y mortalidad materna en México: balances y perspectivas desde la antropología y la interdisciplinariedad. México DF: CIESAS; 2017. p. 168-200.

55. Scopel RPD. A cosmopolítica da gestação, parto e pós-parto: práticas de autoatenção e processo de medicalização entre os índios Munduruku. Brasilia: Paralelo 15; 2015.

56. Ortega Canto J, Palma Ortega J. Comunicación, género y autoatención: acción social en prevención de cánceres. En: Ortega Canto J, Pérez Mutul J, (coords.). Cánceres en mujeres mayas de Yucatán: pobreza, género y comunicación social. México DF: Universidad Autónoma de Yucatán; 2017. p. 199-244.

57. Eroza E, Álvarez Gordillo GC. Narrativas del padecimiento "mental": arenas de debate en torno a prácticas sociales y a los valores que las sustentan entre los mayas tzotziles y tzeltales del 
sur de México. Revista de Antropología Social. 2008;17:377-410.

58. Ponce $P$, Núñez Noriega G. Pueblos indígenas y VIH-SIDA. Desacatos. 2011;(35):7-10.

59. Anderson J, Diez A, Dourojeanni D, Figueroa B, Jiménez O, Miní E, Vallenas S. Mujeres de negro: la muerte materna en zonas rurales del Perú; estudio de casos. Lima: Ministerio de Salud, Proyecto 2000; 1999.

60. Freyermuth Enciso MG. Morir en Chenalhó: género, etnia y generación; factores constitutivos del riesgo durante la maternidad. [Tesis de doctorado]. México DF: Universidad Nacional Autónoma de México; 2000.

61. Sesia P. Quince años de investigaciones en la prevención y la reducción de la muerte materna en Oaxaca: perspectivas y aportes cualicuantitativos desde la antropología y la salud pública. En: Freyermuth Enciso MG, (coord.). Salud y mortalidad materna en México: balances y perspectivas desde la antropología y la interdisciplinariedad. México DF: CIESAS; 2017. p. 202-251.

\section{FORMA DE CITAR}

Menéndez EL. Antropología médica en América Latina 1990-2015: una revisión estrictamente provisional. Salud Colectiva. 2018;14(3):461-481. doi: 10.18294/sc.2018.1838.

Recibido: 17 de abril de 2018 | Versión final: 25 de junio de 2018 | Aprobado: 16 de agosto de 2018

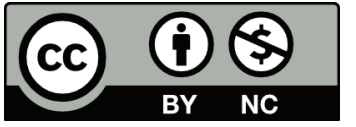

Este obra está bajo una licencia de Creative Commons Reconocimiento-NoComercial 4.0 Internacional. Reconocimiento - Permite copiar, distribuir y comunicar públicamente la obra. A cambio, se debe reconocer y citar al autor original. No Comercial - Esta obra no puede ser utilizada con finalidades comerciales, a menos que se obtenga el permiso. 